\title{
UN ANÁLISIS DE LA EFICIENCIA DE MUNICIPIOS TURÍSTICOS ESPAÑOLES
}

\author{
Elías Bendodo Benasayag* \\ Manuel Ángel Fernández Gámez** \\ José Ramón Sánchez Serrano** \\ Universidad de Málaga
}

\section{RESUMEN}

El objetivo de este estudio es conocer qué factores exógenos condicionan la eficiencia de los municipios con una marcada orientación turística. Este estudio es una de las pocas publicaciones basadas en la eficiencia de municipios turísticos. A diferencia de la mayoría de las investigaciones que evalúan la eficiencia municipal en general, se cree que este documento contribuye a la literatura relacionada ofreciendo ideas a los municipios turísticos relativamente ineficientes sobre la mejora de la eficiencia.

Palabras clave: Eficiencia, Análisis Envolvente de Datos (DEA), Municipios turísticos, España.

\section{An analysis of Spanish Tourism Municipalities efficiency}

\section{ABSTRACT}

The objective of this study is to know which exogenous factors condition the efficiency of the municipalities with tourist orientation. This study is one of the few publications based on the efficiency of tourism municipalities. Unlike most of the research that assesses municipal efficiency in general, it is believed that this document contributes to related literature by offering ideas to relatively inefficient tourism municipalities about improving efficiency. Spain.

Keywords: Efficiency, Data Envelopment Analysis (DEA), Tourism Municipalities,

Fecha de recepción: 22 de noviembre de 2017

Fecha de aceptación: 5 de marzo de 2018

* Programa de Doctorado Interuniversitario en Turismo. Universidad de Málaga.

** Departamento de Finanzas y Contabilidad. Universidad de Málaga. Campus El Ejido s/n. 29071 MÁLAGA (España).E-mail: mangel@uma.es, joseramonsanchez@uma.es 


\section{INTRODUCCION}

El sector turístico es una de las industrias más grandes y de mayor crecimiento en el mundo. Contribuye de manera importante al desarrollo socioeconómico generando empleo, ingresos de exportación e inversiones en infraestructuras. En 2015, los ingresos mundiales por turismo proporcionados por los destinos ascendieron a 1.260 mil millones de dólares, el valor total de las exportaciones de turismo proporcionadas por los servicios de transporte internacional alcanzó los 1,5 billones de dólares, y las llegadas de turistas internacionales fueron de 1.186 millones. La industria turística contribuyó en un 10\% al producto interior bruto (PIB) mundial con respecto a los efectos directos, indirectos e inducidos, y en términos de empleo, contribuyó con uno de cada once empleados (UNWTO, 2016).

En los últimos años, y en el ámbito del sector público, ha habido un interés creciente por introducir medidas para incentivar el desarrollo turístico. El turismo es ya una actividad estratégica en lugares donde esta actividad se presenta como una de las vertientes más prometedoras en su modelo de desarrollo. Incluso todos los ámbitos de responsabilidad política tienen competencias en este desarrollo, abarcando desde el supranacional, el nacional, el regional y el local. En este contexto, las administraciones locales constituyen un papel muy importante en la prestación de servicios públicos en materia de turismo. Se trata de un subsector que con el paso del tiempo ha ido asumiendo más competencias, lo que ha conducido a una acumulación de funciones y, por lo tanto, a un incremento en el gasto y a un mayor peso en la economía. En consecuencia, parece imprescindible adoptar criterios de eficiencia municipal en el momento de gestionar las políticas turísticas, por ejemplo, minimizando costes, buscando oportunidades y definiendo la prioridad de las acciones.

Los estudios sobre la eficiencia respecto a la administración y asignación de los recursos en los municipios es un campo creciente e importante en la literatura (Narbón-Perpiñá y De Witte, 2018; Pérez-López, Prior y Zafra-Gómez, 2015; Selva y Sanz, 2015; Cordero et al., 2016; Storto, 2016; Afonso y Fernandes, 2005). Sin embargo, y hasta donde llega nuestro conocimiento, el análisis sobre las causas de la eficiencia de los municipios turísticos es incipiente y sólo existen algunas experiencias para municipios chinos, demandándose más investigación sobre el tema (Tingting Yi y Liang, 2015).

La literatura existente sobre eficiencia municipal ha concluido, con carácter general, que las diferencias en los niveles de eficiencia son explicadas tanto por factores internos relacionados con el comportamiento de los gestores, como por factores externos o exógenos asociados a las circunstancias geográficas y socioeconómicas (Galariotis et al., 2016; BenitoAccounting, Faculty of Economics and Business, University of Murcia, Campus de Espinardo, Murcia, 30100, Spain Correspondencebenitobl@upct.es, Bastida y Accounting, Technical University of Cartagena , Cartagena, Spain García, 2010; Kalb, 2014; Afonso y Fernández, 2005; Balaguer, Prior y Tortosa, 2007). Los resultados obtenidos indican que los gestores públicos disponen de un margen para optimizar la utilización de los recursos, pero que existe una parte de esas ineficiencias que son debidas a factores exógenos tales como el tamaño de la entidad, la presión fiscal per cápita, los ingresos por transferencias per cápita y el nivel de actividad económica (Arcelus et al., 2007). Por ello, y como 
proponen Seifert y Nieswand (2014), es importante comprender mejor la naturaleza de la ineficiencia debida a factores exógenos con objeto de diseñar políticas que mejoren la asignación de recursos.

Dado que las conclusiones existentes sobre eficiencia municipal están referidas a la generalidad de municipios, y que no existen experiencias para municipios turísticos, en el presente trabajo tratamos de responder a la siguiente cuestión de investigación: Conocer si los factores exógenos que son importantes para la eficiencia municipal en general, también lo son para los municipios con una marcada orientación turística, o por el contrario, en los municipios turísticos inciden factores exógenos diferentes que pueden orientar la implementación de políticas públicas encaminadas a mejorar la eficiencia de dichos municipios.

Con objeto de resolver la cuestión de investigación planteada, el presente trabajo ha seleccionado una muestra integrada por los municipios de la provincia de Málaga. La provincia de Málaga conforma un escenario ideal para nuestro análisis porque presenta algunas particularidades que pueden aportar resultados singulares. En todas las comarcas existe una significativa oferta turística y el sector turístico es el más importante en las economías locales de la provincia (Turismo y Planificación Costa del Sol, 2018). El turismo es el primer sector generador de empleo y riqueza en todos los municipios, que se han convertido en un destino capaz de atraer a más de 9 millones de turistas al año (SOPDE, 2016). La provincia cuenta con 103 municipios, entre los que figuran entidades de diferentes tamaños, con distintos grados de agrupación en la prestación de servicios, sujetos a diversos factores geográficos y socioculturales. A la muestra objeto de estudio se ha aplicado análisis de frontera no paramétrica DEA (Data Envelopment Analysis) para medir la eficiencia municipal. Posteriormente, y con objeto de detectar qué variables exógenas influyen en los niveles de eficiencia, se ha desarrollado un modelo de regresión entre los niveles de eficiencia obtenidos y las hipotéticas variables exógenas.

El trabajo se estructura de la siguiente manera: Tras esta introducción, se realiza una breve revisión de la literatura teórica y empírica acerca del estudio de la eficiencia municipal. A continuación se desarrolla la metodología utilizada. El cuarto apartado presenta los datos utilizados y los resultados alcanzados. Por último, se exponen las principales conclusiones obtenidas y futuras líneas de investigación.

\section{REVISIÓN DE LA LITERATURA}

La eficiencia es la relación entre el producto obtenido y los factores empleados para ello, y se ocupa del rendimiento operacional de una empresa o país (Joppe y Li, 2016). Los procesos que producen más salidas por entrada tienen una mayor eficiencia. Si se proporciona el mayor rendimiento posible por entrada, se logrará una eficiencia óptima. Sin utilizar nuevas tecnologías o hacer varios cambios, es imposible aumentar la eficiencia (Sherman y Zhu, 2006).

Los estudios sobre eficiencia han tenido su origen en los trabajos de Farrell (1957) y Charnes, Cooper y Rhodes (1978). Posteriormente se han desarrollado numerosos trabajos, abarcando desde el ámbito privado al sector público, que han resaltado la eficiencia como elemento importante para la expansión de la producción y del progreso técnico, y 
como condición necesaria para la formulación de políticas económicas coherentes con necesidades reales de las entidades (Ayaviri y Alarcón, 2014).

Con el aumento de los servicios públicos ofrecidos a nivel local y de las restricciones presupuestarias y financieras a los gobiernos locales, ha crecido la necesidad de buscar nuevas formas de gestión de los servicios públicos locales, a fin de maximizar la eficiencia en la utilización de los recursos. La literatura existente sobre eficiencia municipal no ha sido ajena a este problema y ha abordado el análisis desde diversos puntos de vista. De una manera global (Storto, 2016; Selva y Sanz, 2015; Ferreira y Cunha, 2014; Balaguer, 2004; De Borger et al, 1994; Giménez y Prior, 2003; Prieto y Zofio, 2001; Taïrou, 2000; Vanden Eeckaut, Tulkens y Jamar, 1993; Worthington, 2000), y con base en la prestación de un único servicio (Bosch, Pedraja y Suárez, 2000). Además, para el análisis de la eficiencia se han aplicado diversos métodos, entre los que destacan tanto modelos paramétricos y no paramétricos (Drew y Dollery, 2015; Selva y Sanz, 2015; Doumpos y Cohen, 2014; Balaguer, Prior y Vela, 2003; Balaguer, 2004; Moore, Nolan y Segal, 2005) como modelos determinísticos y estocásticos (Lovell, 2000; Afonso y Fernandes, 2005). Esta medición de la eficiencia municipal se ha venido realizando, a su vez, bajo dos enfoques diferentes (input y output), cuya elección depende de cuáles sean las variables sobre las que un gobierno local tiene la capacidad de decisión (De Borger y Kerstens, 1996). En este contexto, la mayor parte de los trabajos empíricos tienden a orientarse desde el enfoque input, pues se considera que el output viene determinado por una situación institucional que uniformiza los bienes y servicios públicos a proveer (Arcelus et al., 2007).

Sobre los factores que justifican las diferencias en los niveles de eficiencia municipal, las conclusiones de la investigación previa señalan tanto factores internos relacionados con el comportamiento de los gestores, como factores externos o exógenos asociados a las circunstancias geográficas y socioeconómicas (Galariotis et al., 2016; BenitoAccounting, Faculty of Economics and Business, University of Murcia, Campus de Espinardo, Murcia, 30100, Spain Correspondencebenitobl@upct.es, Bastida y Accounting, Technical University of Cartagena , Cartagena, Spain García, 2010; Kalb, 2014; Afonso y Fernandes, 2005; Balaguer, Prior y Tortosa, 2006; De Borger y Kerstens, 1996; Thomas y Callan, 2001; Selva y Sanz, 2015). Con objeto de delimitar los dos ámbitos de análisis se vienen utilizando, a su vez, dos métodos (Arcelus et al., 2007). Uno de ellos consiste en seleccionar el conjunto de variables de input o output que se consideran discrecionales por parte de los gestores municipales y, posteriormente, realizar la maximización respecto de este conjunto. El otro método se realiza en dos etapas, en primer lugar obteniendo la frontera en función de los inputs que se consideran bajo el control del decisor, y a continuación, realizando una regresión entre los niveles de eficiencia obtenidos y los factores fuera de control, para encontrar los factores externos o exógenos que puedan afectar a la eficiencia.

En la literatura previa se encuentra un cierto consenso acerca de los factores externos o exógenos que afectan a la eficiencia municipal, sobre todo en referencia al esfuerzo fiscal, al tamaño del municipio, al efecto de la mancomunación, y al desarrollo económico.

El esfuerzo fiscal y la recaudación por transferencias han sido utilizados habitualmente como variables exógenas. BenitoAccounting, Faculty of Economics and Business, University of Murcia, Campus de Espinardo, Murcia, 30100, Spain Correspondencebenitobl@ upct.es, Bastida y Accounting, Technical University of Cartagena , Cartagena, Spain Gar- 
cía (2010), en referencia a municipios españoles, han concluido que cuanto mayor sea la carga fiscal, mayor será la eficiencia en la prestación de servicios. Por su parte, Arcelus et al. (2007) estudiaron una muestra de municipios de Navarra, y confirmaron que cuando los recursos municipales provienen más de los tributos propios que de transferencias, la eficiencia es mayor, debido a que los contribuyentes son más sensibles al gasto que se realice. Kalb (2014), en un estudio sobre eficiencia municipal en Alemania, indica que los ingresos disponibles de los ciudadanos y las subvenciones intergubernamentales influyen negativamente en la eficiencia. Por su parte, Monkam (2014) indica que la autonomía fiscal influye en la eficiencia productiva de los municipios africanos. Kalseth y Rattso (1998) y Borge, Falch y Tovmo (2004), en referencia a administraciones locales de Noruega, analizaron el impacto de las instituciones políticas y presupuestarias sobre la eficiencia en la producción de servicios. De Borger y Kerstens (1996), con una muestra de municipios Belgas, demostraron que los municipios con alta capacidad para obtener recursos por recaudación o por transferencias se encuentran menos motivados para ser gestionados de forma eficiente. Worthington (2000) realiza un análisis similar para los municipios australianos.

El impacto del tamaño del municipio y la distancia a la capital nacional también han sido identificados como causas externas de ineficiencia municipal en numerosas investigaciones (Seifert y Nieswand, 2014; Thomas y Callan, 2001; Díez-Ticio y Mancebón, 2003). Por ejemplo, Balaguer, Prior y Tortosa (2004) analizaron la relación entre eficiencia y grado de descentralización, señalando que a mayor número de habitantes mayor es el número de servicios prestados $\mathrm{y}$, por tanto, que hay una relación positiva entre grado de descentralización y eficiencia municipal. Igualmente, Sousa y Stosic (2005) utilizan métodos alternativos para explicar la relación entre la eficiencia y el tamaño de los municipios. Recientemente, Selva y Sanz (2015), con una muestra de municipios de Castilla-La Mancha, llegan a la conclusión de que el nivel de eficiencia aumenta a medida que lo hace el tamaño de la entidad, y que la eficiencia es mayor en los municipios cuya población está menos dispersa. No obstante lo anterior, también observaron la presencia de economías de escala hasta un determinado número de habitantes, o bien una débil asociación entre ambas variables.

Otros resultados relevantes de la literatura apuntan al efecto de la mancomunación como un claro factor de eficiencia en el coste de producción de los servicios prestados por los municipios. Galariotis et al. (2016) destacaron el efecto positivo de dicha mancomunación en municipios franceses. Arcelus et al. (2007), con una muestra de municipios de Navarra, detectan un alto nivel de significatividad del fenómeno de agrupación de servicios, aunque consideran que los mayores niveles de eficiencia municipal pueden atribuirse, además de a la propia mancomunación, al potencial para hacerlo que implica la proximidad u otros factores que lo permitan.

Finalmente, los niveles de desarrollo económico del municipio también han sido objeto de estudio como factores exógenos de eficiencia. Por ejemplo, Balaguer, Prior y Vela (2003), estudiando municipios valencianos, concluyeron que parte de las ineficiencias observadas son debidas a factores exógenos tales como el nivel de actividad económica. En general, las conclusiones de la investigación previa apuntan a que municipios con un alto desarrollo económico tienden a recaudar más ingresos, por lo que relajan la gestión 
y son menos eficientes (De Borges y Kerstens, 1996). Sin embargo, otros estudios no han observado un impacto significativo del nivel económico sobre el grado de eficiencia local (Giménez y Prior, 2003; Benito et al., 2010).

Como se ha expuesto, son numerosos los trabajos que han investigado sobre los factores exógenos que determinan las causas de las ineficiencias municipales. Sin embargo, son prácticamente inexistentes los que obtienen conclusiones para municipios turísticos, y los trabajos que han relacionado la actividad turística y la eficiencia se han limitado casi exclusivamente al ámbito de unidades estatales o regionales. En este sentido, Herrero-Prieto y Gómez-Vega (2017) analizaron la eficiencia de las regiones españolas utilizando los recursos culturales como un atractivo turístico. Soysal-Kurt (2017) midió la eficiencia turística de los países europeos. Por su parte, Cracolici et al. (2008) estudiaron la eficiencia de determinadas regiones italianas y concluyeron que la ineficiencia de las regiones turísticas estaba condicionada por la regulación de la actividad, las características físico-geográficas y la cultura. Tingting Yi y Liang (2015) estudiaron la evolución de la eficiencia de determinados municipios y provincias chinas, pero no llegaron a identificar los factores exógenos que condicionaban la eficiencia municipal. Finalmente, otros trabajos también han aportado determinadas evidencias acerca de la relación entre eficiencia de las administraciones públicas y desarrollo turístico, aunque con controversia. Por ejemplo, Giménez y Prior (2007) piensan que la eficiencia es mayor en aquellos municipios en los que existe más actividad turística, y que esta eficiencia viene moderada por el tamaño de los municipios. Balaguer-Coll, Prior-Jiménez y Vela-Bargues (2003) indican que el nivel turístico es una variable socio-económica que no afecta de forma significativa al nivel de eficiencia de dichas administraciones públicas. Y por su parte, Kalb, Geys y Heinemann (2012), para una muestra de municipios alemanes, sostienen que ignorar la influencia de los factores exógenos conduce a un sesgo significativo en los niveles de eficiencia estimados, sobre todo en municipios de alta actividad turística.

\section{MÉTODOS}

Entre las técnicas no paramétricas para el análisis de la eficiencia destaca DEA. Las primeras mediciones de eficiencia según la metodología DEA fueron propuestas por Farrell (1957) y por Charnes, Cooper y Rhodes (1978), que utilizaron un enfoque de programación matemática en la construcción de las fronteras de producción con una orientación input, asumiendo la hipótesis de rendimientos constantes a escala (CRS). La orientación input busca, dado el nivel de outputs, la máxima reducción proporcional en el vector de inputs mientras permanece en la frontera de posibilidades de producción. De este modo, una unidad de producción no es eficiente si es posible disminuir cualquier input sin alterar sus outputs. Por su parte, la hipótesis CRS hace referencia a los rendimientos a escala que caracterizan la tecnología de producción, es decir, a la forma en que los factores productivos (inputs) se combinan para obtener los productos (outputs), y que en este caso se suponen obtienen rendimientos a escala constantes.

En el análisis DEA, la frontera permite ajustar en una envolvente los datos tomando como referencia las unidades más eficientes. De este modo, el desempeño de cualquier 
unidad analizada se realiza en comparación con las de mejor eficiencia (Albi, 1992). DEA se sustenta en la idea de que si una unidad de producción puede lograr un determinado nivel de output a partir de un nivel de inputs, todos las demás unidades están en condiciones de hacer lo mismo o tomar medidas que le permitan mejorar su eficiencia (Figura 1).

\section{Figura 1 \\ FRONTERA EFICIENTE (ORIENTACIÓN INPUT)}

En la figura, la frontera de eficiencia viene representada por la isocuanta unitaria SS'. Las unidades técnicamente eficientes son A y B, mientras que la unidad C es ineficiente. La medición de la eficiencia técnica corresponde a la razón OR/OC. Se trata de un modelo con dos inputs $\left(\mathrm{x}_{1} \mathrm{y}_{2}\right)$ y un solo output $(\mathrm{y})$.

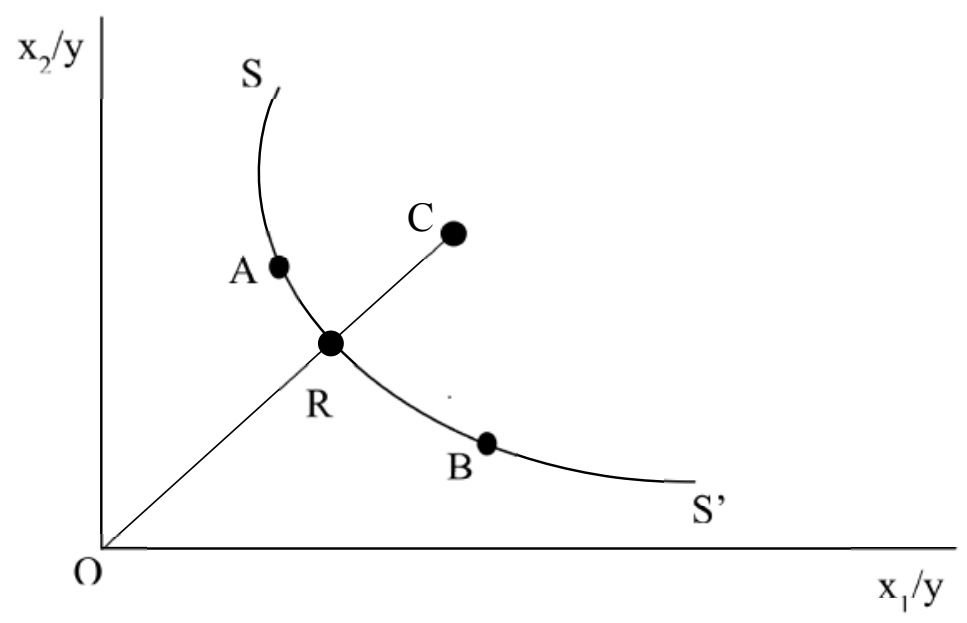

Fuente: Elaboración propia a partir de Farrell (1957)

DEA ha sido utilizado recientemente en el análisis de la eficiencia de entidades locales, usando diferentes inputs y outputs. Por ejemplo, Doumpos y Cohen (2014) para medir la eficiencia de municipios griegos, Drew y Dollery (2015) y Worthington (2000) para municipios australianos. Prieto y Zofio (2001), con una muestra de pequeños municipios de Castilla-León, usaron como inputs los gastos presupuestados y como outputs el abastecimiento de agua, las infraestructuras medioambientales, la urbanización y el equipamiento cultural. Taïrou (2000) utilizó DEA para analizar la eficiencia de municipios franceses. Balaguer (2004), también con metodología DEA, analizó una muestra de 258 municipios de la Comunidad Valenciana utilizando cuatro inputs y siete outputs, entre los que se incluyen aquéllos que hacen referencia a la calidad de los servicios prestados.

Nuestro análisis utiliza una orientación input para medir la eficiencia (Balaguer, Prior y Vela, 2003), según el cual, la eficiencia de cada entidad o unidad está caracterizada por su habilidad para alcanzar un determinado nivel de output utilizando la menor cantidad 
posible de inputs. El enfoque DEA bajo la hipótesis CRS (Charnes, Cooper y Rhodes, 1978), se formula como aparece en (1).

$\operatorname{Min} \Theta_{(\lambda, \theta)}(1)$

sujeto a las restricciones detalladas en (2).

$$
\begin{aligned}
& x_{i} \Theta-X \lambda \geq 0 \\
& Y \lambda-y_{r} \geq 0(2) \\
& =1 \\
& \lambda \geq 0
\end{aligned}
$$

donde $\theta$ indica la distancia en inputs a la envolvente de datos, es decir, la medida de eficiencia $X$ es la matriz de inputs de orden $(m \times n), Y$ es la matriz de outputs de orden $(s \mathrm{x} n), \lambda$ es el vector $(n \mathrm{x} l)$ de pesos o intensidades, y $x_{i}$ e $y_{r}$ representan los vectores de inputs y outputs, respectivamente.

Una vez obtenidos los resultados de la eficiencia, corresponde aplicarlos al análisis de regresión para conocer la dependencia entre estos resultados de eficiencia y las variables explicativas. A tal fin se utilizan variables exógenas con las que se intentan comprobar si la eficiencia está influenciada por el grado de agrupación en la prestación de los servicios o mancomunación, por la presión fiscal, por factores geográficos, por factores sociales y por el desarrollo económico de los municipios. Para ello, utilizamos una regresión truncada de tipo "bootstrapped". Esta regresión permite una inferencia válida en esta segunda etapa evitando las limitaciones que imponen otras alternativas de modelización (Banker y Natarajan, 2008; Simar y Wilson, 2007). En consecuencia, el modelo de análisis adopta la estructura expresada en (3).

$$
E F_{\mathrm{j}}=\beta_{0}+\beta_{j} Z_{j}+\mathrm{e}_{j}(3)
$$

siendo $E F_{j}$ el vector de observaciones de eficiencia, $\beta_{o}$ el término constante, $Z_{j}$ el vector de observaciones de las variables exógenas específicas de cada municipio, $\beta_{j}$ los coeficientes de dichas variables exógenas, y e $_{j}$ el término de error de la regresión, que es restringido por la condición (4).

$$
\mathrm{e}_{j} \leq 1-\beta_{0}-\beta_{j} Z_{j}(4)
$$

Como proponen Simar y Wilson (2007), se asume que este término e $_{j}$ sigue una distribución normal truncada con media cero (antes del truncamiento), varianza desconocida y un punto de truncamiento determinado por esta condición. Y dado que el verdadero $E F$ es desconocido, se reemplaza con los valores de eficiencia obtenidos en la primera etapa 
(). De este modo, la ecuación (3) se transforma en (5), y debe ser resuelta por MáximaVerosimilitud respecto a $\beta_{j}$ y a $\sigma$.

$$
\widehat{E F_{l}}=\beta_{0}+\beta_{j} Z_{j}+\mathrm{e}_{j} \text { siendo } \mathrm{e} \sim \mathrm{N}\left(0, \sigma^{2}\right) \text { tal que } \mathrm{e}_{j} \leq 1-\beta_{0}-\beta_{j} Z_{j}(5)
$$

Dado que nuestro propósito es determinar si la eficiencia de los municipios de la muestra $(E F)$ está influenciada por las variables exógenas seleccionadas conforme a la literatura existente, utilizaremos el modelo que aparece en (5), pero especificado según (6).

$$
E F_{i}=\beta_{0}+\beta_{1} M C_{j}+\beta_{2} P F_{j}+\beta_{3} F G_{j}+\beta_{4} F S_{j}+\beta_{5} D E_{j}+\beta_{6} G A_{j}+T A_{j}+\mathrm{e}_{j}(6)
$$

donde $E F_{j}$ es la variable dependiente de eficiencia calculada con DEA. Por su parte, $M C_{j}$ representa el grado de agrupación en la prestación de servicios y es captado a través de una variable nominal que toma el valor 1 se la entidad es miembro de una mancomunidad de municipios y 0 en caso contrario. $P F_{j}$ es un índice de presión fiscal obtenido del cociente entre los capítulos económicos 1 al 3 y la suma de capítulos económicos 1 al 5 . $F G_{j}$ hace referencia al factor geográfico, tomando el valor 0 si la entidad es un municipio de interior y 1 si es costero. $F S_{j}$ representa la incidencia de factores sociales utilizando como proxy el número de subsidios de desempleo. $D E_{j}, G A_{j}$ y $T A_{j}$ captan la incidencia de los factores de desarrollo económico. La primera, en relación con el número de establecimientos comerciales. El resto, para captar otras circunstancias económicas: $G A_{j}$ toma el valor 1 si el municipio está considerado legalmente de gran afluencia turística y 0 en caso contrario). Una zona de gran afluencia turística es cualquier parte o el todo de un municipio que por una o diversas circunstancias de interés económico, durante todo el año, o parte de él, justifica un tratamiento excepcional en cuanto a los horarios y días de apertura de su oferta comercial a los consumidores de ese municipio. Según la normativa andaluza, se entiende por zonas de gran afluencia turística los términos municipales o parte de los mismos en los que, en determinados períodos del año, la media ponderada anual de población sea significativamente superior al número de residentes, o en los que tenga lugar una gran afluencia de visitantes por motivos turísticos. $T A_{j}$ toma el valor 1 si el municipio está declarado legalmente como Municipio Turístico de Andalucía y 0 en caso contrario. La declaración de Municipio Turístico de Andalucía queda regulada por el Decreto 72/2017, de 13 de junio, para dar respuesta a los problemas con los que se encuentran algunos municipios andaluces como consecuencia de los flujos de turistas que reciben a lo largo del año. Con dicha declaración, la Junta de Andalucía estará obligada a considerar al municipio de forma prioritaria en las acciones de ordenación y fomento de sus planes económicos cuando resulte compatible con los objetivos de los mismos y en su caso, con el carácter finalista de su financiación.

Finalmente, e representa el error residual.

\section{MUESTRA Y DATOS}

Para estimar la frontera de producción, y exigiendo que los resultados sean consistentes, hemos utilizado la información de una muestra integrada por los 103 municipios de la provincia de Málaga, referida al período 2010-2015. 
La consecución de los objetivos de la presente investigación ha requerido combinar diversas fuentes estadísticas sobre variables financieras y sobre variables económicas de los municipios analizados. Ambos tipos de variables son necesarias para conocer los niveles de eficiencia de los municipios y para determinar sus principales conductores. La no existencia de una base de datos sobre municipios españoles que combine datos financieros con variables económicas nos ha obligado a la construcción de la misma a partir de las fuentes que relacionamos a continuación.

Las variables financieras han sido obtenidas del Tribunal de Cuentas de España (2016). En el caso de las variables económicas se han utilizado, a su vez, diferentes fuentes. Por un lado, la Encuesta de Infraestructura y Equipamientos Locales del Ministerio de Hacienda y Administraciones Públicas para la información referente a factores del ámbito físico (población, superficie vías urbanas y suelo urbano), a la calidad de las infraestructuras (información de carácter categórico que hace referencia a una calidad buena, regular o mala, y que hemos ponderado por el volumen de servicios prestados por cada municipio) y al ámbito del desarrollo económico (establecimientos comerciales, plazas hoteleras, instalaciones deportivas y centros culturales). Por otro lado, el Boletín Oficial de la Junta de Andalucía (BOJA número 27 de 7 de enero de 2013) para la calificación de municipio de Gran Afluencia Turística y el Decreto 72/2017, de 13 de junio (BOJA número 119 de 23 de junio de 2017) para la declaración de Municipio Turístico de Andalucía.

Finalmente, el Sistema de Calidad Turística de la Secretaría de Estado de Turismo (SICTED) para identificar a los municipios que ostentan el distintivo Compromiso de Calidad Turística. La figura 2 detalla la situación de cada municipio de la muestra respecto a la calificación de Gran Afluencia Turística, SICTED y Municipio Turístico de Andalucía.

Figura 2

\section{CALIFICACIÓN TURÍSTICA DE LOS MUNICIPIOS DE LA MUESTRA}

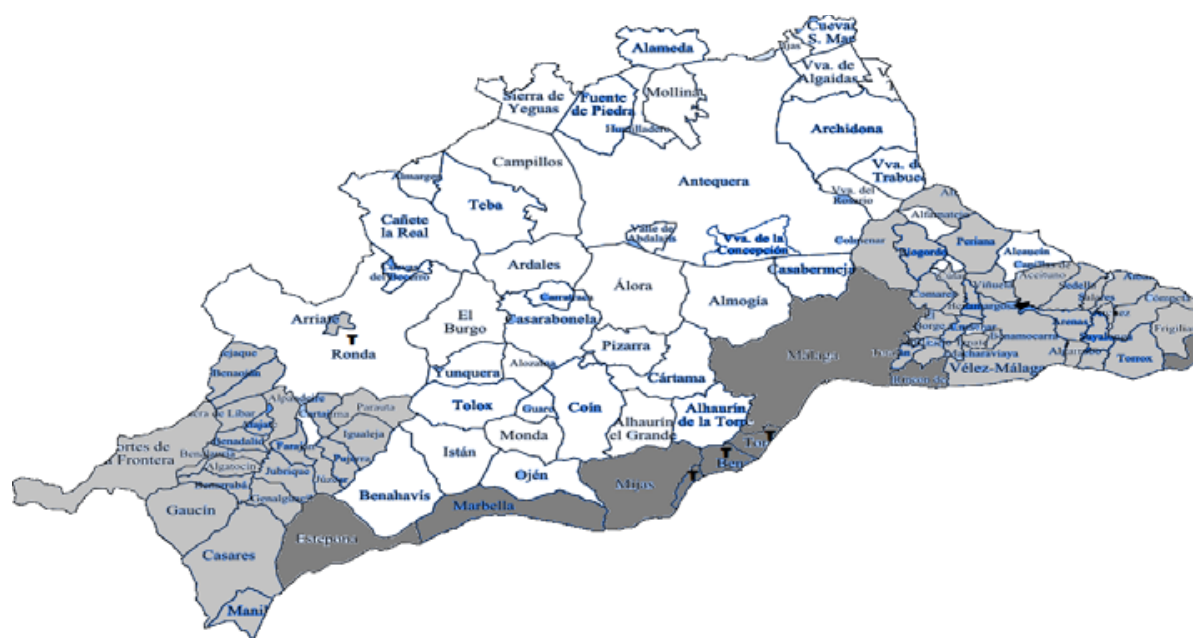

Municipio con SICTED (62) Municipio con SICTED y de Gran Afluencia Turística (9) T Municipio Turístico de Andalucía (5) 
Para construir el indicador DEA de la eficiencia de los municipios de la muestra hemos seleccionado los inputs y outputs utilizados en la literatura previa. Como inputs se han empleado los datos presupuestarios correspondientes a los capítulos 1 al 7 (exceptuando el capítulo 3 de gastos financieros). Estos indicadores han sido usados por Arcelus et al. (2007), Worthington (2000), Giménez y Prior (2003), Taïrou (2000) y Balaguer (2004). Y como outputs se han utilizado un conjunto de indicadores que pretenden representar los servicios que prestan los municipios. En este caso, los representativos de la dimensión poblacional de los servicios (población total), del ámbito físico de prestación (superficie urbana y longitud de vías urbanas), del nivel de calidad de los servicios (calidad de las infraestructuras) y del ámbito económico (número de plazas hoteleras, de instalaciones deportivas y de centros culturales). Los citados outputs de las dimensiones poblacional, de ámbito físico y de calidad de los servicios han sido utilizados previamente por Balaguer (2004), De Borger y Kerstens (1996), Taïrou (2000), y Vanden Eeckaut, Tulkens, y Jamar (1993). La tabla 1 muestra la relación detallada de los inputs y outputs seleccionados.

\section{Tabla 1 \\ DEFINICIÓN DE INPUTS Y OUTPUTS DE EFICIENCIA}

\section{Indicadores de inputs}

Capítulo 1 de gastos (remuneraciones al personal)

Capítulo 2 de gastos (compra de bienes y servicios)

Capítulos 4 y 7 de gastos (transferencias corrientes y de capital, respectivamente)

Capítulo 6 de gastos (inversiones reales)

Indicadores de outputs

Población total

Dimensión poblacional

Superficie urbana $\left(\mathrm{km}^{2}\right)$

Ámbito físico

Longitud vías urbanas $\left(\mathrm{m}^{2}\right)$

Calidad de los servicios

Calidad de las infraestructuras (ponderación de la calidad de las vías urbanas, de iluminación, de parques y del suministro de agua)

Número de plazas hoteleras

Ámbito de desarrollo económico

Superficie de instalaciones deportivas $\left(\mathrm{m}^{2}\right)$

Superficie de centros culturales $\left(\mathrm{m}^{2}\right)$ 


\subsection{Análisis descriptivo}

En la tabla 2 aparecen los principales descriptivos de los inputs y outputs de eficiencia de la muestra, segmentada según el Compromiso de Calidad Turística de los municipios (SICTED). Para el total de la muestra, los valores medios de remuneraciones al personal ascienden a 7.046.715,74 euros, los de compra de bienes y servicios a 4.940.410,21 euros, los correspondientes a transferencias corrientes y de capital a 1.933.940,73 euros y los de inversiones reales a 2.056.798,69 euros. No obstante, los anteriores registros son sensiblemente inferiores en el segmento de municipios sin SICTED. Además, la muestra se caracteriza por un número medio de habitantes de $16.128,45$ y una superficie urbana de $71,53 \mathrm{~km}^{2}$. Por su parte, la longitud media de vías urbanas es de 72.206,52 metros y el nivel ponderado de calidad de las infraestructuras asciende a 35.174,12. También se caracteriza por un número medio de plazas hoteleras cercano a 1.000 plazas y una superficie media de instalaciones deportivas y culturales de $34.891,73 \mathrm{~m}^{2}$ y $7.921,78 \mathrm{~m}^{2}$, respectivamente. En la segmentación de la muestra, los municipios con SICTED poseen mayor número de habitantes, mayor longitud de vías urbanas y un mayor número medio de plazas hoteleras. Sin embargo, tienen una menor superficie urbana y una menor calidad de infraestructuras.

Tabla 2

DESCRIPTIVOS DE INPUTS Y OUTPUTS DE EFICIENCIA

\begin{tabular}{|c|c|c|c|c|}
\hline Variables & Unidades & Media & Mediana & Desv. Estándar \\
\hline \multicolumn{5}{|l|}{$\begin{array}{l}\text { a) Total municipios } \\
(n=103)\end{array}$} \\
\hline \multicolumn{5}{|l|}{ Inputs } \\
\hline Capítulo 1 & $\begin{array}{c}\text { Remuneraciones al personal } \\
(€)\end{array}$ & 7046715,74 & 973762,00 & 23690576,25 \\
\hline Capítulo 2 & $\begin{array}{l}\text { Compra de bienes y } \\
\text { servicios }(€)\end{array}$ & 4940410,21 & 671681,00 & 18985205,47 \\
\hline Capítulos 4 y 7 & $\begin{array}{c}\text { Transf. corrientes y de } \\
\text { capital }(€)\end{array}$ & 1933940,73 & 169515,00 & 8001666,94 \\
\hline Capítulo 6 & Inversiones reales $(€)$ & 2056798,69 & 677608,00 & 5575396,72 \\
\hline \multicolumn{5}{|l|}{ Outputs } \\
\hline Población & $\mathrm{N}^{\mathrm{o}}$ de habitantes & 16128,45 & 2527,00 & 59902,99 \\
\hline Superficie & Superficie urbana $\left(\mathrm{km}^{2}\right)$ & 71,53 & 40,00 & 95,94 \\
\hline Vías urbanas & Longitud vías urbanas (m) & 72206,52 & 16354,00 & 187813,15 \\
\hline Calidad servicios & Calidad infraestructuras & 35174,12 & 12813,65 & 55904,24 \\
\hline Plazas hoteleras & $\mathrm{N}^{\mathrm{o}}$ de plazas hoteleras & 964,72 & 44,00 & 3263,671 \\
\hline Inst. deportivas & $\begin{array}{l}\text { Superficie de inst. } \\
\text { deportivas }\left(\mathrm{m}^{2}\right)\end{array}$ & 34891,73 & 15321,24 & 71700,75 \\
\hline $\begin{array}{l}\text { Centros } \\
\text { culturales }\end{array}$ & $\begin{array}{l}\text { Superficie de c. culturales } \\
\qquad\left(\mathrm{m}^{2}\right)\end{array}$ & 7921,78 & 1883,56 & 24534,86 \\
\hline
\end{tabular}




\begin{tabular}{|c|c|c|c|c|}
\hline Variables & Unidades & Media & Mediana & Desv. Estándar \\
\hline \multicolumn{5}{|c|}{ b) Municipios con SICTED (n=62) } \\
\hline \multicolumn{5}{|c|}{ Inputs } \\
\hline Capítulo 1 & $\begin{array}{c}\text { Remuneraciones al personal } \\
(€)\end{array}$ & 10116847,95 & 614512,00 & 30332650,63 \\
\hline Capítulo 2 & $\begin{array}{l}\text { Compra de bienes y } \\
\text { servicios }(€)\end{array}$ & 7153399,98 & 456034,50 & 24391582,30 \\
\hline Capítulos 4 y 7 & $\begin{array}{c}\text { Transf. corrientes y de } \\
\text { capital }(€)\end{array}$ & 2943428,03 & 131778,50 & 10273806,80 \\
\hline Capítulo 6 & Inversiones reales $(€)$ & 2650436,85 & 638955,00 & 7103408,80 \\
\hline \multicolumn{5}{|l|}{ Outputs } \\
\hline Población & $\mathrm{N}^{\mathrm{o}}$ de habitantes & 22425,95 & 1509,00 & 76950,10 \\
\hline Superficie & Superficie urbana $\left(\mathrm{km}^{2}\right)$ & 53,80 & 29,85 & 76,07 \\
\hline Vías urbanas & Longitud vías urbanas (m) & 94960,70 & 10810,50 & 238992,98 \\
\hline Calidad servicios & Calidad infraestructuras & 20220,52 & 5906,61 & 41704,45 \\
\hline Plazas hoteleras & $\mathrm{N}^{\mathrm{o}}$ de plazas hoteleras & 1523,50 & 25,50 & 4147,50 \\
\hline Inst. deportivas & $\begin{array}{l}\text { Superficie de inst. } \\
\text { deportivas }\left(\mathrm{m}^{2}\right)\end{array}$ & 34092,39 & 8429,86 & 73575,33 \\
\hline $\begin{array}{l}\text { Centros } \\
\text { culturales }\end{array}$ & $\begin{array}{l}\text { Superficie de c. culturales } \\
\qquad\left(\mathrm{m}^{2}\right)\end{array}$ & 8693,21 & 1046,34 & 30244,22 \\
\hline \multicolumn{5}{|c|}{ c) Municipios sin SICTED $(n=41)$} \\
\hline \multicolumn{5}{|l|}{ Inputs } \\
\hline Capítulo 1 & $\begin{array}{c}\text { Remuneraciones al personal } \\
(€)\end{array}$ & 2553839,34 & 1155214,00 & 3343135,37 \\
\hline Capítulo 2 & $\begin{array}{c}\text { Compra de bienes y } \\
\text { servicios }(€)\end{array}$ & 1701888,60 & 786435,00 & 2334236,62 \\
\hline Capítulos 4 y 7 & $\begin{array}{c}\text { Transf. corrientes y de } \\
\text { capital }(€)\end{array}$ & 456642,24 & 199298,00 & 783093,23 \\
\hline Capítulo 6 & Inversiones reales $(€)$ & 1188059,92 & 765826,00 & 1408642,29 \\
\hline \multicolumn{5}{|l|}{ Outputs } \\
\hline Población & $\mathrm{N}^{\mathrm{o}}$ de habitantes & 6912,59 & 3364,00 & 9509,75 \\
\hline Superficie & Superficie urbana $\left(\mathrm{km}^{2}\right)$ & 97,49 & 73,10 & 115,39 \\
\hline Vías urbanas & Longitud vías urbanas (m) & 38907,73 & 21364,00 & 44975,10 \\
\hline Calidad servicios & Calidad infraestructuras & 57057,42 & 27548,03 & 66494,61 \\
\hline Plazas hoteleras & $\mathrm{N}^{\mathrm{o}}$ de plazas hoteleras & 147,00 & 74,00 & 320,09 \\
\hline Inst. deportivas & $\begin{array}{l}\text { Superficie de inst. } \\
\text { deportivas }\left(\mathrm{m}^{2}\right)\end{array}$ & 36061,50 & 19165,12 & 69752,93 \\
\hline $\begin{array}{l}\text { Centros } \\
\text { culturales }\end{array}$ & $\begin{array}{l}\text { Superficie de c. culturales } \\
\left(\mathrm{m}^{2}\right)\end{array}$ & 6792,86 & 2128,64 & 12389,44 \\
\hline
\end{tabular}

La tabla 3 y la figura 3 detallan la distribución de los valores de eficiencia obtenidos. Para los municipios con SICTED existe una estructura tri-modal, lo que indica que se presentan tres grupos de municipios: Los que son eficientes, aquellos que son algo eficientes, y los que son ineficientes. Para los que no poseen SICTED, por el contrario, se 
observa una estructura bi-modal, diferenciando claramente entre municipios eficientes e ineficientes. La comparación de dichas estructuras con la que presenta la muestra total de municipios permite inferir el efecto de la calidad turística, que parece moderar la ineficiencia municipal.

Tabla 3

PRINCIPALES ESTADÍSTICOS DE LA EFICIENCIA ESTIMADA

\begin{tabular}{lccccccc}
\hline & Media & Mediana & Mínimo & Máximo & Desv. Est. & $\begin{array}{c}\text { Unidades } \\
\text { eficientes }\end{array}$ & $\begin{array}{c}\% \\
\text { eficientes }\end{array}$ \\
\hline $\begin{array}{l}\text { Municipios con } \\
\text { SICTED }\end{array}$ & 0,752 & 0,704 & 0,387 & 1 & 0,279 & 17 & 27,41 \\
$\begin{array}{l}\text { Municipios sin } \\
\text { SICTED }\end{array}$ & 0,648 & 0,735 & 0,391 & 1 & 0,280 & 19 & 46,34 \\
\begin{tabular}{l} 
Total municipios \\
\hline
\end{tabular} & 0,719 & 0,683 & 0,391 & 1 & 0,296 & 18 & 17,47 \\
\hline
\end{tabular}

Figura 3

FUNCIONES DE DENSIDAD DE LA EFICIENCIA

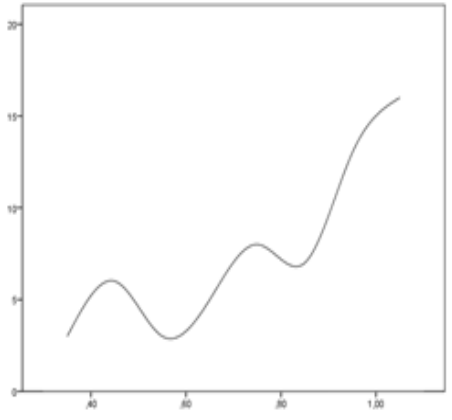

Municipios con SICTED
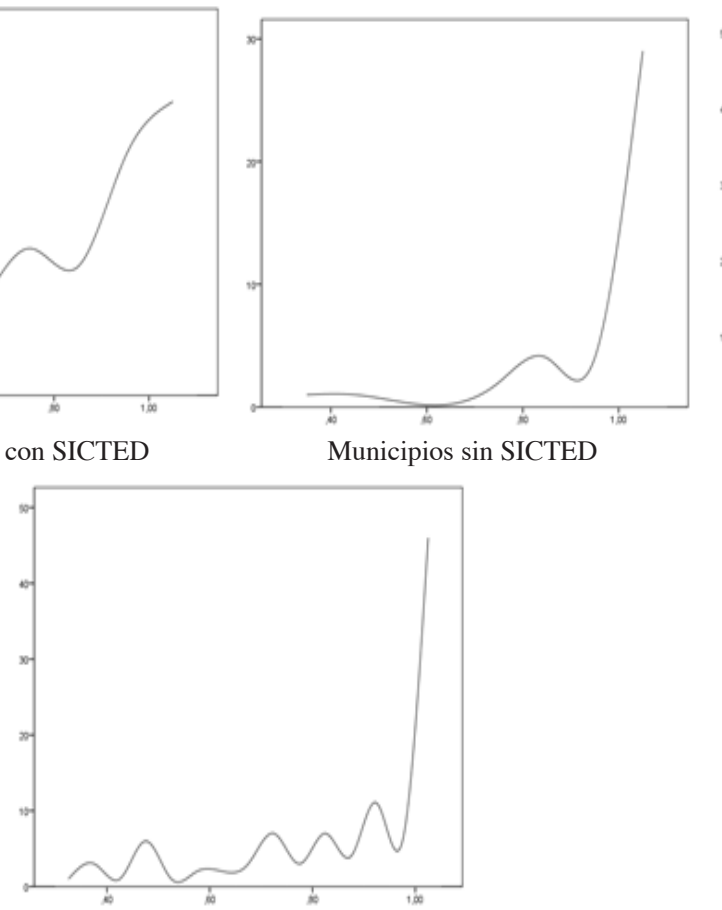

Total municipios 
Por su parte, las variables independientes seleccionadas, distintas de las utilizadas para el cálculo de la eficiencia, y que hipotetizamos como factores exógenos, se basaban en dos criterios: el estudio de la literatura y los datos disponibles. Las características de dichas variables aparecen en la tabla 4. Para el total de la muestra, el índice de presión fiscal (PF) arroja un valor medio de 0,88 , el número de subsidios de desempleo asciende a 426 y el número medio de establecimientos comerciales es de 354. Además, el 50,48\% de los municipios están agrupados en mancomunidad y un 13,59\% son costeros. En la tabla 4 también puede comprobarse que los municipios con SICTED están sometidos a una menor presión fiscal, pero que soportan mayores niveles de subsidios de desempleo y poseen mayor número de establecimientos comerciales. Así mismo, se caracterizan por estar más agrupados en mancomunidades y por su localización costera.

Tabla 4

DESCRIPTIVOS DE LAS VARIABLES INDEPENDIENTES

\begin{tabular}{|c|c|c|c|c|}
\hline Variables & Unidades & Media & Mediana & Desv. Estándar \\
\hline \multicolumn{5}{|c|}{ a) Total municipios $(n=103)$} \\
\hline $\mathrm{PF}$ & Cap. 1 al 3/ Cap. 1 al 5 & 0,88 & 0,90 & 0,13 \\
\hline FS & $\mathrm{N}^{\mathrm{o}}$ de subsidios de desempleo & 426,43 & 89,00 & 1420,72 \\
\hline $\mathrm{DE}$ & $\begin{array}{l}\mathrm{N}^{\circ} \text { de establecimientos } \\
\text { comerciales }\end{array}$ & 354,42 & 41,00 & 1402,07 \\
\hline
\end{tabular}

MC Municipios en mancomunidad: 50,48\%

FC Municipios costeros: 13,59\%

GA Municipios de gran afluencia: 8,73\%

TA Municipios Turísticos de Andalucía: 4,85\%

b) Municipios con SICTED $(n=62)$

$\begin{array}{llccc}\text { PF } & \text { Cap. 1 al 3/ Cap. 1 al 5 } & 0,86 & 0,90 & 0,17 \\ \text { FS } & N^{o} \text { de subsidios de desempleo } & 557,39 & 60,50 & 1814,27 \\ \text { DE } & \begin{array}{l}N^{o} \text { de establecimientos } \\ \text { comerciales }\end{array} & 503,58 & 22,50 & 1801,92\end{array}$

MC Municipios en mancomunidad: 59,68\%

FC Municipios costeros: 22,58\%

GA Municipios de gran afluencia: $14,52 \%$

TA Municipios Turísticos de Andalucía: 6,45\%

c) Municipios sin SICTED $(n=41)$

$\begin{array}{llccc}\text { PF } & \text { Cap. 1 al 3/ Cap. 1 al 5 } & 0,90 & 0,90 & 0,04 \\ \text { FS } & N^{o} \text { de subsidios de desempleo } & 228,39 & 152,00 & 246,08 \\ \text { DE } & N^{o} \text { de establecimientos } & 136,12 & 60,00 & 207,70\end{array}$

MC Municipios en mancomunidad: 29,27\%

FC Municipios costeros: $0,00 \%$

GA Municipios de gran afluencia: $0,00 \%$

TA Municipios Turísticos de Andalucía: 2,44\% 


\subsection{Estimación de resultados}

Para la resolución de la ecuación (6) hemos realizado una regresión truncada de tipo "bootstrapped" que nos informa sobre la relación entre eficiencia $\left(E F_{j}\right)$ y las variables independientes seleccionadas. Los resultados de la regresión aparecen en la tabla 5, en la que el modelo 1 hace referencia a la muestra total de municipios, el modelo 2 a la muestra de municipios con SICTED y el modelo 3 a los municipios sin SICTED.

Tabla 5

REGRESIÓN (ECUACIÓN 6; VARIABLE DEPENDIENTE: EFICIENCIA)

\begin{tabular}{|c|c|c|c|}
\hline Variable & Modelo 1 & Modelo 2 & Modelo 3 \\
\hline Constante & $\begin{array}{l}0,40 * * \\
(0,00)\end{array}$ & $\begin{array}{l}0,42 * * \\
(0,00)\end{array}$ & $\begin{array}{l}-1,01^{*} \\
(0,04)\end{array}$ \\
\hline $\mathrm{MC}$ & $\begin{array}{l}0,28 * * \\
(0,00)\end{array}$ & $\begin{array}{l}0,29 * * \\
(0,00)\end{array}$ & $\begin{array}{l}0,31 * * \\
(0,00)\end{array}$ \\
\hline $\mathrm{PF}$ & $\begin{array}{c}0,18 \\
(0,45)\end{array}$ & $\begin{array}{c}0,13 \\
(0,16)\end{array}$ & $\begin{array}{l}1,68 * * \\
(0,00)\end{array}$ \\
\hline FG & $\begin{array}{l}-0,02 \\
(0,79)\end{array}$ & $\begin{array}{l}-0,01 \\
(0,81)\end{array}$ & - \\
\hline FS & $\begin{array}{c}0,02 \\
(0,59)\end{array}$ & $\begin{array}{c}0,03 \\
(0,82)\end{array}$ & $\begin{array}{c}0,01 \\
(0,16)\end{array}$ \\
\hline $\mathrm{DE}$ & $\begin{array}{r}-0,04 \\
(0,53)\end{array}$ & $\begin{array}{l}-0,02 \\
(0,74)\end{array}$ & $\begin{array}{c}0,01 \\
(0,38)\end{array}$ \\
\hline GA & $\begin{array}{l}0,11 * \\
(0,03)\end{array}$ & $\begin{array}{l}0,11^{*} \\
(0,04)\end{array}$ & - \\
\hline $\mathrm{TA}$ & $\begin{array}{l}0,10^{*} \\
(0,03)\end{array}$ & $\begin{array}{l}0,09 * \\
(0,04)\end{array}$ & $\begin{array}{c}0,03 \\
(0,83)\end{array}$ \\
\hline $\mathrm{N}^{\mathrm{o}}$ de observaciones & 103 & 62 & 41 \\
\hline $\mathrm{F}$ & $\begin{array}{c}23,42 * * \\
(0,00)\end{array}$ & $\begin{array}{c}19,65 * * \\
(0,00)\end{array}$ & $\begin{array}{c}10,62 * * \\
(0,00)\end{array}$ \\
\hline $\mathrm{R}^{2}$ Nagelkerkel & 0,68 & 0,79 & 0,65 \\
\hline Durbin-Watson & 2,16 & 2,05 & 2,03 \\
\hline
\end{tabular}

**Coeficientes significativos a un nivel de significación de 0.01 ; Coeficientes significativos a un nivel de significación de 0.05; Nivel de significación entre paréntesis.

Los resultados de la tabla 5 indican que el coeficiente estimado asociado a la variable explicativa $M C$-que mide el grado de mancomunación de los municipios- es positivo y estadísticamente significativo en los tres modelos desarrollados ( $\mathrm{p}$-valor $<0,01$ ). Por tanto, existe una relación directa entre los niveles de mancomunación y la eficiencia munici- 
pal. Además, los resultados de regresión también indican que la variable relativa a la presión fiscal $(P F)$ es significativa y está en relación directa con la eficiencia municipal (p-valor $<0,01$ ), pero sólo en el segmento de municipios sin SICTED (modelo 3). Por su parte, las variables de desarrollo económico $G A$ y $T A$, que hace referencia a la situación de gran afluencia y de declaración de Municipio Turístico de Andalucía, han resultado significativas en los modelos 1 y 2 , reflejando su relación directa (positiva) respecto a los niveles de eficiencia de los municipios (p-valor $<0,05)$. Igualmente, los resultados reflejan que los factores geográficos $(F G)$, los factores sociales $(F S)$ y algún aspecto del desarrollo económico $(D E)$ no tienen una significativa relación con la eficiencia de los municipios.

Las relaciones detectadas entre la eficiencia y las variables exógenas analizadas permiten conocer aspectos de gran importancia sobre la ineficiencia que presentan los municipios turísticos de la muestra. De una parte, la importancia de la mancomunación de los servicios, de la calificación de zona de gran afluencia y de la declaración de Municipio Turístico de Andalucía. La ausencia de la agrupación de los servicios prestados, la falta de libertad de horarios comerciales que marca la regulación de municipio de gran afluencia, y la prioridad en las acciones de ordenación y fomento de planes económicos que promueve dicha declaración de Municipio Turístico de Andalucía están, pues, condicionando la eficiencia municipal. De otra parte, el efecto moderador de la calidad de los servicios municipales en el impacto de la presión fiscal sobre la eficiencia, pues en aquellos municipios turísticos con compromiso de calidad turística (SICTED), la presión fiscal no está relacionada con dicha eficiencia.

Comparando los resultados anteriores con los obtenidos por la literatura previa en relación con los factores exógenos que condicionan la eficiencia municipal, pero para municipios no especialmente turísticos, detectamos que la significación de la variable de agrupación en mancomunidades es acorde con las conclusiones generales sobre eficiencia municipal. Los trabajos previos de Galariotis et al. (2016), Afonso y Fernandez (2005), Balaguer, Prior y Tortosa (2006) y Arcelus et al. (2007) confirman el efecto de la mancomunación como un claro factor de eficiencia en el coste de producción de los servicios prestados por los municipios, y en nuestra muestra de municipios turísticos también se ha verificado. Por su parte, y en referencia a los efectos de la presión fiscal, nuestros resultados difieren de los obtenidos para la generalidad de municipios en la literatura previa. Monkam (2014), BenitoAccounting, Faculty of Economics and Business, University of Murcia, Campus de Espinardo, Murcia, 30100, Spain Correspondencebenitobl@upct. es, Bastida y Accounting, Technical University of Cartagena , Cartagena, Spain García (2010), y Arcelus et al. (2007) concluyeron que cuanto mayor es la carga fiscal, mayor resulta la eficiencia municipal. Sin embargo, el presente trabajo ha constatado que, en municipios turísticos, dicho efecto afecta a la eficiencia sólo cuando los municipios no ostentan compromiso de calidad turística (SICTED). Por tanto, sólo en esta categoría de municipios turísticos se demuestra que una alta capacidad para obtener recursos por recaudación motiva más para ser gestionados de forma eficiente. De otro lado, y en referencia al desarrollo económico, ya aludimos a la controversia existente en la literatura previa, pues mientras De Borges y Kerstens (1996) piensan que municipios con un alto desarrollo económico tienden a recaudar más ingresos y por tanto relajan la gestión y son menos eficientes, otros estudios no han observado un impacto significativo del nivel de desarrollo 
económico sobre el grado de eficiencia local (Giménez y Prior, 2003; BenitoAccounting, Faculty of Economics and Business, University of Murcia, Campus de Espinardo, Murcia, 30100, Spain Correspondencebenitobl@upct.es, Bastida y Accounting, Technical University of Cartagena , Cartagena, Spain García, 2010). En nuestro estudio, sin embargo, hemos podido constatar que el desarrollo económico medido a través de GA y TA está en relación directa con la eficiencia municipal. De este modo, también podemos comprender cómo una mayor libertad de horarios comerciales y una prioridad en las acciones de ordenación y fomento de los planes económicos municipales están asociadas a una mayor eficiencia, o lo que sería lo mismo, que la ausencia de medidas que incentivan el desarrollo de la actividad económica frena la posibilidad de un mayor nivel de eficiencia de los municipios turísticos. Finalmente, y conforme a los resultados obtenidos para el resto de variables independientes, hemos constatado también que los factores geográficos $(F G)$ y los factores sociales $(F S)$ no tienen una significativa influencia en la eficiencia de los municipios turísticos, lo que difiere de las conclusiones obtenidas por Seifert y Nieswand (2014), Thomas y Callan (2001), Díez-Ticio y Mancebón (2003) y Balaguer, Prior y Vela (2003) en relación con municipios sin especial orientación turística.

\section{CONCLUSIONES}

El presente trabajo amplía la investigación desarrollada recientemente sobre la eficiencia municipal aportando conocimiento sobre aquellas entidades que poseen una especial orientación turística. Con objeto de determinar si los factores exógenos que son importantes para la eficiencia municipal en general, también lo son para los municipios con una marcada orientación turística, y exigiendo que los resultados sean consistentes, hemos utilizado información financiera, económica, geográfica y social del período 2010-2015 de una muestra integrada por los 103 municipios pertenecientes a la provincia de Málaga. El modelo de análisis estimado explica la eficiencia de los municipios turísticos en función de los niveles de mancomunación, de la presión fiscal, de los factores geográficos, de los factores sociales y del desarrollo económico.

Conforme a los resultados obtenidos, hemos podido constatar que en el ámbito turístico municipal existen ineficiencias y que éstas son debidas en parte a factores exógenos, concretamente, al grado de mancomunación, a la presión fiscal y al nivel de desarrollo económico. El fenómeno de la mancomunación de servicios ha resultado significativo para explicar la eficiencia de los municipios de especial orientación turística. La agrupación en mancomunidades ha resultado asociada a una mayor eficiencia municipal. Destaca igualmente, por su novedad en el análisis, el efecto positivo que sobre la eficiencia han manifestado las variables de desarrollo económico que hace referencia a aquellos municipios con gran afluencia turística y a los declarados como Municipios Turísticos de Andalucía. Por tanto, un tratamiento excepcional en cuanto a los horarios y días de apertura de la oferta comercial a los consumidores y turistas, y acciones prioritarias en cuanto a la ordenación y a planes económicos ha quedado asociado a una mayor eficiencia municipal.

Por su parte, los resultados obtenidos sobre la variable presión fiscal nos han permitido entender que, en determinados municipios, una mayor presencia de recursos tributarios frente a transferencias corrientes y/o ingresos patrimoniales está asociada a mayores nive- 
les de eficiencia. Concretamente, esta relación ha resultado significativa sólo en los municipios no acogidos al Sistema de Calidad Turística (SICTED). Parece, pues, que un nivel de calidad homogéneo en los servicios ofrecidos al turista tiene un efecto moderador en las repercusiones de la presión fiscal sobre la eficiencia municipal. Estos resultados podrían explicarse por el marco de sensibilidad que surge cuando las empresas y los servicios turísticos públicos y privados están adheridos un plan de calidad, en el que se desarrollan propuestas de mejoras del destino que culminan motivando a una gestión más eficiente.

Dado los efectos que determinados factores exógenos han mostrado en los modelos construidos, entendemos que la gestión de las políticas económicas en entornos turísticos necesariamente ha de adoptar criterios de eficiencia, minimizando costes, buscando oportunidades y definiendo prioridad en las acciones. Las administraciones han de ser conscientes, además, de que determinados factores exógenos tienen un impacto positivo en la eficiencia de los municipios turísticos y que, por tanto, deben fomentar medidas en materia fiscal, de comercio y de calidad adaptadas a las expectativas del consumidor. Futuras líneas de investigación podrían extender nuestras conclusiones a otros entornos turísticos nacionales e internacionales. También focalizando en la eficiencia municipal en referencia a la prestación de un único servicio, o explorando nuevos métodos de análisis que ayuden a incrementar el poder explicativo de los modelos.

\section{REFERENCIAS}

AFONSO, A. y FERNANDES, S. (2005): Public Services Efficiency Provision in Italian regions: a non parametric analysis, ISEG-UTL Economics Working Paper, No.2/2005/ DE/CISEP, Technical University of Lisbon. Disponible en http://dx.doi.org/10.2139/ ssrn.670741

ALBI,E. (1992): "Evaluación de la eficiencia pública. El control de la eficiencia del sector público", Hacienda Pública Española, no 120, pp. 299-319.

ARCELUS, F.J., AROCENA, P., CABASÉS, F. y PASCUAL, P. (2007): Eficiencia municipal en Navarra: Efectos de la mancomunación de servicios. Santander, XIV Encuentro de Economía Pública, pp. 1-22.

AYAVIRI, D. y ALARCÓN, S. (2014): "Clasificación económica de los municipios de Bolivia", Perspectivas, $\mathrm{n}^{\circ}$ 33, pp. 29-55.

BALAGUER, M.T. (2004): "La eficiencia en las administraciones locales ante diferentes especificaciones del output", Hacienda Pública Española, nº 170 (3), pp. 37-58.

BALAGUER, M.T., PRIOR, D. y TORTOSA, E. (2006): Decentralization and efficiency in Spanish local government. IVIE Working Paper, EC 2006-02, pp. 1-33.

BALAGUER, M.T., PRIOR, D. y TORTOSA, E. (2007): "On the determinants of local government performance: A two-stage nonparametric approach". European Economic Review, vol. 51 (2), pp. 425-451.

BALAGUER, M.T., PRIOR, D. y VELA, J.M. (2003): Eficiencia y calidad en la gestión de las Administraciones Locales. Santa Cruz de Tenerife, $X$ Encuentro de Economía Pública.

BANKER, R.D. y NATARAJAN, R. (2008): "Evaluating Contextual Variables Affecting Productivity Using Data Envelopment Analysis", Operations Research, vol. 56 (1), pp. 48-58. 
BENITOAccounting, Faculty of Economics and Business, University of Murcia, Campus de Espinardo, Murcia, 30100, Spain Correspondencebenitobl@upct.es, B., BASTIDA, F. y Accounting, Technical University of Cartagena , Cartagena, Spain GARCÍA, J.A. (2010): "Explaining differences in efficiency: an application to Spanish municipalities", Journal Applied Economics, vol. 42 (4), pp. 515-528.

BORGE, L.E., FALCH, T. y TOVMO, P. (2004): Efficiency in Public Service Production: The Impact of Political and Budgetary Institutions. W.P. Department of Economics, Norwegian University of Science and Technology, pp. 1-22.

BOSCH, N., PEDRAJA; F., y SUÁREZ, J. (2000): "Measuring the Efficiency in Spanish Municipal Refuse Collection services”, Local Government Studies, vol. 26 (3), pp. 71-90.

CHARNES, A., COOPER, W.W. y RHODES, E. (1978): "Measuring the Efficiency of Decisions Making Units”, European Journal of Operational Research, vol. 2 (6), pp. 429-444.

CORDERO, J.M., PEDRAJA-CHAPARRO, F., PISAFLORES, E.C. y POLO, C. (2016): "Efficiency assessment of Portuguese municipalities using a conditional nonparametric approach", Journal of Productivity Analysis, vol. 48 (1), pp. 1-24.

CRACOLICI, M.F., NIJKAMP, P. y RIETVELD, P. (2008): “Assessment of tourism competitiveness by analysing destination efficiency", Tourism Economics. vol. 14 (2), pp. 325-342.

DE BORGER, B. y KERSTENS, K. (1996): "Radial and Nonradial Measurement of Technical Efficiency: An Empirical Illustration for Belgian Local Governments using an FDH Reference Technology", Journal of Productivity Analysis, vol. 7 (1), pp. 41-62.

DE BORGER, B., KERSTENS, K., MOESEN, W. y VANNESTE, J. (1994): “Explaining differences in productive efficiency: An application to Belgian municipalities", Public Choice, vol. 80 (3-4), pp. 339-358.

DÍEZ-TICIO, M.A. y MANCEBÓN, M.J. (2003): “Análisis de la eficiencia de las instituciones encargadas de la seguridad ciudadana: algunas enseñanzas procedentes del ámbito de la economía del delito", Papeles de Economía Española, nº 95, pp. 306-319.

DOUMPOS, M. y COHEN, S. (2014): “Applying data envelopment analysis on accounting data to assess and optimize the efficiency of Greek local governments", Omega, vol. 46, pp. 74-85.

DREW, J. y DOLLERY, B. (2015): "The State of Things: The Dynamic Efficiency of Australian State and Territories", Economic Papers: A journal of applied economics and policy, vol. 34 (3), pp. 165-176.

FARRELL, M.J. (1957): "The Measurement of Productive Efficiency", Journal of the Royal Statistical Society Series A, vol. 120 (3), pp. 45-67.

FERREIRA, N. y CUNHA, R. (2014): "Revisiting the determinants of local government performance", Omega, vol. 44, pp. 91-103.

GALARIOTIS, E., GUYOT, A., DOUMPOS, M. y ZOPOUNIDIS, C. (2016): “A novel multiattribute benchmarking approach for assessing the financial performance of local governments: Empirical evidence from France", European Journal of Operational Research, vol. 248 (1), pp. 301-317. 
GIMÉNEZ, V.M. y PRIOR, D. (2003): "Evaluación frontera de la eficiencia en costes. Una aplicación a los ayuntamientos de Cataluña", Papeles de Economía Española, n ${ }^{\circ}$ 95, pp. 113-124.

GIMÉNEZ, V.M., y PRIOR, D. (2007): “Long-and short-term cost efficiency frontier evaluation: evidence from Spanish local governments", Fiscal Studies, vol. 28 (1), pp. 121-139.

HERRERO-PRIETO, L.C., y GÓMEZ-VEGA, M. (2017): “Cultural resources as a factor in cultural tourism attraction Technical efficiency estimation of regional destinations in Spain", Tourism Economics, vol. 23 (2), pp. 260-280.Principio del formulario

Final del formulario

JOPPE, M. y LI, X.P. (2016): "Productivity measurement in tourism: The need for better tools", Journal of Tourism Research, vol. 55 (2), pp. 139-149.

KALB, A. (2014): "What Determines Local Governments' Costefficiency? The Case of Road Maintenance", Regional Studies, vol. 48 (9), pp. 1483-1498.

KALB, A., GEYS, B. y HEINEMANN, F. (2012): "Value for money? German local government efficiency in a comparative perspective", Applied Economics, vol. 44 (2), pp. 201-218.

KALSETH, J. y RATTSO, J. (1998): "Political control of administrative spending: The case of local governments in Norway", Economics and Politics, vol. 10 (1), pp. 63-83.

LOVELL, C. (2000): "Measuring Efficiency in the Public Sector", en Blank, Jos L.T., (Ed.), Public Provision and Performance. New York, Oxford University Press, pp. 3-67.

MONKAM, N.F. (2014): "Local municipality productive efficiency and its determinants in South Africa", Development Southern Africa, vol. 31 (2), pp. 275-298.

MOORE, A., NOLAN, J. y SEGAL, G.F. (2005): "Putting Out the Trash. Measuring Municipal Service Efficiency in U.S. Cities", Urban Affairs Review, vol. 41 (2), pp. 237-259.

NARBÓN-PERPIÑÁ, I. y DE WITTE, K. (2017): “Local governments’ efficiency: A systematic literature review-part I". International Transactions in Operational Research, vol. 25 (2), pp. 431-468.

PÉREZ-LÓPEZ, G., PRIOR, D., y ZAFRA-GÓMEZ, J.L. (2015): “Rethinking New Public Management Delivery Forms and Efficiency: Long-Term Effects in Spanish Local Government", Journal of Public Administration Research and Theory, vol. 25(4), pp. 1157-1183.

PRIETO, A.M. y ZOFÍO, J.L. (2001): "Evaluating Effectiveness in Public Provision of Infrastructure and Equipment: The Case of Spanish Municipalities", Journal of Productivity Analysis, vol. 15 (1), pp. 41-58.

SEIFERT, S., y NIESWAND, M. (2014): “What Drives Intermediate Local Governments' Spending Efficiency: The Case of French Départements", Local Government Studies, vol. 40 (5), pp. 766-790.

SELVA, C. y SANZ, M.M. (2015): "La eficiencia municipal en la prestación de bienes y servicios públicos: De la medición de su nivel al análisis de sus posibles causas", ICE Tribuna de Economía, no 882, pp. 157-169. 
SHERMAN, H.D. y ZHU, J. (2006): Service productivity management: Improving service performance using data envelopment analysis. Boston, MA: Springer.

SIMAR, L. y WILSON, P.W. (2007): "Estimation and inference in two-stage, semiparametric models of production processes", Journal of Econometrics, vol. 136 (1) pp. 31-64.

SOPDE (2016): Turismo y Planificación Costa del Sol. Disponible en http://www.costadelsolmalaga.org/

SOUSA M. y STOSIC, B. (2005): “Technical Efficiency of the Brazilian Municipalities: Correcting Nonparametric Frontier Measurements for Outliers", Journal of Productivity Analysis, vol. 24 (2), pp. 157-181.

SOYSAL-KURT, H. (2017): "Measuring Tourism Efficiency of European Countries by Using Data Envelopment Analysis”, European Scientific Journal, vol. 13 (10), pp. 1857-1881.

STORTO, C. (2016): "The trade-off between cost efficiency and public service quality: A non-parametric frontier analysis of Italian major municipalities", Cities, vol. 51, pp.

TAIIROU, A.A. (2000): “Does inefficiency explain financia! vulnerability of French municipalities?". International Conference on Accounting, Auditing \& Management in Public Sector Reforms. Zaragoza: EIASM, pp. 589-599.

THOMAS, J.M. y CALLAN, S.J. (2001): "Economies of Scale and Scope: A Cost Analysis of Municipal Solid Waste Services", Land Economics, vol. 77 (4), pp. 548-560.

TINGTING YI, T., y LIANG, M. (2015): "Evolutional Model of Tourism Efficiency Based on the DEA Method: A Case Study of Cities in Guangdong Province, China", Asia Pacific Journal of Tourism Research, vol. 20 (7), pp. 789-806.

TRIBUNAL DE CUENTAS DE ESPAÑA (2016): Consulta de Entidades y Cuentas. Disponible en http://www.rendiciondecuentas.es

TURISMO Y PLANIFICACIÓN COSTA DEL SOL (2018): Fortalezas del turismo de interior. Disponible en www.costadelsolmalaga.org

UNWTO (2016): Tourism highlights 2016 Edition.

WORTHINGTON, A.C. (2000): "Cost efficiency in Australian Local Government: A comparative analysis of mathematical programming and econometric approaches", Financial Accountability Management, vol. 16 (3), pp. 201-224.

VANDEN EECKAUT, P., TULKENS, H. y JAMAR, M. A. (1993): "Cost efficiency in Belgian municipalities" en Fried, Lovell \& Schimidt (Eds.): The Measuring of Productive Efficiency. Techniques and Applications, pp. 300-324. 